\title{
The Long-distance Reconfiguration Design of FPGA Based on ARM
}

\author{
Chun Zeng*
}

Chongqing Technology and Business Institute, Chongqing, P.R. China

\begin{abstract}
The task of this design is using the developing embedded technique, ARM to configurate FPGA, so that the reconfiguration of FPGA can without dedicated configuration software, and the configuration of FPGA can no longer to connect with a computer. Save the configuration file in the memory chip, connect the ARM system to the Internet and remote access service to download configuration file for the configuration of FPGA. In the later period of this design, process the FPGA contiguration file(format conversion, encrypt, decode, etc.), and popularize this method to different series of FPGA. So that, the reconfiguration of FPGA do not need to connect FPGA with a computer, only need to get the configuration file. In this paper, structure NFS file system of ARM and computer is succeed, so does the wired communication and the process of configuration file, and the SP1 communication between ARM and FPGA for configuration is achieved.
\end{abstract}

Keywords: ARM, embedded Linux, FPGA, reconfigurable technology.

\section{INTRODUCTION}

Embedded system was born in the microcomputer age in the 1970s [1], the customization of it was transiently explored by researchers, and then the independent embedded system microcontroller began to be studied, that is to add peripheral integrated circuit technique on the original processor, and combine both of them to develop into SCM (Single Chip Microcomputer) with processor kernel, thereby develop into intellectualized microcontroller electronic system.

The primary mission of this design is to make the FPGA reconfiguration away from configuration software, and make the FPGA configuration without being connected with the computer directly, using ARM to process the reconfiguration of FPGA. The configuration files generated while the laboratory design FPGA system can be processed after the configuration file format is studied clearly (format, encryption, decryption, etc.), and the file processing method can be promoted to various series FPGA of this type. In this way, as long as there is a configuration file the FPGA can be deployed, and there is no need to connect FPGA system with experimental computer. The FPGA system can download configuration file through the network of itself when added with wireless modules, and reconfigure it or carry on dynamic reconfiguration.

\section{THE CONCEPTUAL DESIGN OF THE TOPIC}

\subsection{The Selection of ARM Development Board}

ARM processor is a simplify instruction set computer system designed by ARM company of the UK, which is mainly for low budget market. ARM processor is also called the Acorn RISC Machine in the past. As a 32-bit processor, the instruction set of ARM is 32 bit, but it is also compatible with 16 bit Thumb instruction set [2]. The economy of code can achieve $35 \%$ by using the Thumb instruction set than using 32-bit codes in the same condition in general, but most advantages of 32-bit processor system can still be kept.

At present, the ARM microprocessor has been widely used in different fields:

1. Wireless communication field: ARM has the characteristics of low cost and high performance, so it has occupied more and more market share in the field of infinite communication.

2. Network applications: Broadband devices with the ARM technology are more and more competitive in the network application. In addition, the ARM Company have been carrying on new optimization for audio and video processing in the new ARM chips, and promote it vigorously, the traditional application fields of DSP which adopt ARM kernel chips are also more competitive.

3. Consumer electronics: the mobile Internet has been developing swift; the application of ARM technology on mobile Internet terminal devices is broadened.

4. Imaging and security products: ARM Company design SecurCore series ARM chips which have been widely used dedicated to the application areas which have higher safety requirements, ARM technology processing chips are widely used in the current popular digital camera.

5. Industrial control area: ARM series chips also have a wide application in the field of industrial control.

There are 31 general-purpose registers and six status registers in ARM, these registers are not access in all conditions, there are seven different kinds of ARM processor working modes, working in different modes corresponding to different registers. However, there are parts of the registers are 
universal to different processor working modes; the rest of the registers have their corresponding physical registers in different work modes. ARM microprocessors are various, and can provide much different architecture according to different application fields. Among them, some architecture does not contain MMU, as to unable to support WinCE and standard Linux. To the types without MMU units, such as ARM7TDMI hardware platform, operating system uCLinux can be used with no need of MMU support, etc. In fact, there are a variety of microprocessors without MMU units which have successfully transplanted uCLinux, and have good performance in stability and other aspects.

\subsection{S3C2440 Chip Performance Parameters}

S3C2440A is a microprocessor pushed out by Samsung Semiconductor Corp., which has the characteristics of high performance, low power consumption, high integration, and it has gone through the industrial-grade EMC test. It is a microprocessor with industrial temperature range and performance. S3 C2440A has the following advantages:

1. Main frequency is up to $533 \mathrm{MHZ}$.

2. The temperature range is wide including the industrial temperature range.

3. Support more LCD screen resolution.

4. Low operating voltage in the kernel.

5. there are a large number of available UO ports.

6. There are many different kinds of supported voltage memories.

7. Codec Ports meet with AC9'7 specification.

8. Digital camera interface.

9. Each of the input and output of UART port of chip has a capacity of 64 bytes FIFO.

10. Touchscreen interface doesn't need peripheral circuit.

\subsection{Choice of the FPGA Development Board}

FPGA, namely the Field Programmable Gate Array, is further developed on the basis of the GAL (Generic Array Logic), CPLD (Complex Programmable Logic Device), and other programmable devices. FPGA is a kind of half-custom circuits, used for the field of application-specific integrated circuit; FPGA not only solved the problem which is the lack of custom circuits, and overcome the shortcomings of the limitation of the number of original programmable device gate.

The algorithm structure of FFT (Fast Fourier transform)on FPGA is :

$$
\begin{aligned}
& X(\mathrm{k})=\sum_{n=0}^{N-1} x(\mathrm{n}) \mathrm{W}_{N}^{n k} \\
& W_{N}=\exp (-\mathrm{j} 2 \pi / N) \mathrm{k}=0,1 \ldots \ldots, \quad \mathrm{N}-1
\end{aligned}
$$

When the FPGA device is charging, the data stored in the EPROM connected with the FPGA chip will be read in the chip select RAM programming, after the configuration is complete, the FPGA will enter the working condition configured by the configuration file. When the power is shut down, the FPGA internal logical relationship disappears, and return to the white piece, therefore, the FPGA can be used repeatedly. Programming of FPGA device doesn't need to use dedicated FPGA programming, only generic EPROM and PROM programmer need to be used. If you need to modify the function of FPGA, just write data to an EPROM, or to change a piece of EPROM data that has been written. In this way, a piece of FPGA chip can be made to produce different circuit function by using different programming data.

Main technical features of FPGA of Spartan-3E series are shown in Table 1.

The main characteristic of Spartan-3E are: using $90 \mathrm{craft}$; has a large number of I/O ports of users, supports $376 \mathrm{I} / \mathrm{O}$ port or 156 ports; The transmission rate of single-ended port can reaches 622, and DDR interface is supported; Port voltage can be set according to the specific requirements at $3.3 \mathrm{~V}$, $2.5 \mathrm{~V}, 1.8 \mathrm{~V}, 1.5 \mathrm{~V}$ or $1.2 \mathrm{~V} .36$ special multipliers, 231 pieces of distributed RAM, 648 pieces of RAM are maximal supported; there are wide clock frequency and multiple digital clock management (DCM) modules of dedicated chips.

\subsection{Choice of Operating System}

All kinds of operating systems can be adopted on embedded system, so an operating system that is suitable for project development according to different hardware and specific tasks should be chosen. As to the CPU without MMU (Memory Management Unit), uClinux operating system

Table 1. The main technical parameters of Spartan-3E.

\begin{tabular}{|c|c|c|c|c|c|c|c|c|}
\hline Type & $\begin{array}{c}\text { System gate } \\
\text { number }\end{array}$ & $\begin{array}{c}\text { SLICE } \\
\text { number }\end{array}$ & $\begin{array}{c}\text { Distributive } \\
\text { RAM capacity }\end{array}$ & $\begin{array}{c}\text { Block RAM } \\
\text { capacity }\end{array}$ & $\begin{array}{c}\text { Dedicated } \\
\text { mutipliers } \\
\text { number }\end{array}$ & $\begin{array}{c}\text { DCM } \\
\text { number }\end{array}$ & $\begin{array}{c}\text { Max usable } \\
\text { IO number }\end{array}$ & $\begin{array}{c}\text { Check IO } \\
\text { logarithmic }\end{array}$ \\
\hline \hline XC3S100E & $100 \mathrm{~K}$ & 960 & $15 \mathrm{~K}$ & $72 \mathrm{~K}$ & 4 & 2 & 108 & 40 \\
\hline $\mathrm{XC} 3 \mathrm{~S} 250 \mathrm{E}$ & $250 \mathrm{~K}$ & 2448 & $38 \mathrm{~K}$ & $216 \mathrm{~K}$ & 12 & 4 & 172 & 68 \\
\hline $\mathrm{XC} 3 \mathrm{~S} 500 \mathrm{E}$ & $500 \mathrm{~K}$ & 4656 & $73 \mathrm{~K}$ & $360 \mathrm{~K}$ & 20 & 4 & 232 & 92 \\
\hline $\mathrm{XC} 3 \mathrm{~S} 1200 \mathrm{E}$ & $1200 \mathrm{~K}$ & 8672 & $136 \mathrm{~K}$ & $504 \mathrm{~K}$ & 28 & 8 & 304 & 124 \\
\hline $\mathrm{XC} 3 \mathrm{~S} 1600 \mathrm{E}$ & $1500 \mathrm{~K}$ & 14752 & $231 \mathrm{~K}$ & $648 \mathrm{~K}$ & 36 & 8 & 376 & 156 \\
\hline
\end{tabular}


should be used instead of embedded Linux. And embedded Linux can be used as to relative high-end hardware with MMU units. In order to avoid a variety of difficulties when transplant operating system into specific hardware environment, and meanwhile accelerate the development schedule of whole design, the embedded operating systems that have high portability should be chosen.

\subsection{Windows CE Compared with Linux}

The relative advantages of Windows CE than Linux:

1. The development of it difficulty is low, and it is easy to be transplanted, the Windows application program under the development of PC can run under Windows CE through simple transplant. The introduction to the Linux is too difficult, and the operations are often achieved with commands, as the engineers who are familiar with Linux are too little and the integrated development environment is poor [3].

2. Developing specifications, due to that the source code Linux is open and free, anyone can add and modification into it, which results in the poor compatibility of products based on Linux, and Windows CE is set by the Microsoft completely, which does not allow outsiders to modify, and the compatibility of product is good.

3. The debugging is simple and convenient, Windows $\mathrm{CE}$ provided debugging methods based on the serial port, USB and Ethernet interface, Such as providing the ActiveSync debugging software through Microsoft, and downloading system image files, debugging applications, monitoring all kinds of content of targets easily through USB.

4. Highly modular design, the users can cut the modules provided necessarily, to generate a fully functional, small capacity kernel that meets the demands of particular devices.

5. To support multiple hardware platforms, Windows CE support the mainstream embedded CPUs as X86, ARM, and MIPS, SuperH, etc.

6. Powerful development tools, Windows CE provide developers with the powerful function, friendly interface development tools, such as kernel compilation used by the Platform Builde to complete, and the compiling and debugging of application program completed by using $\mathrm{EV} \mathrm{C}++$.

7. Rich multimedia support, technologies based on DirectX API and the Windows Media can provide high performance Audio and video and 3D image processing.

The relative disadvantages of Windows CE than Linux:

1. The source code of Linux is open, many Linux enthusiasts around the world are in research and exploration on it, they are the powerful technology supporters of the developers; while the code of Windows CE is only part open to the public.

2. The Linux source code is free and competitive in price, a lot of manufacturers choose free Linux operation system as software platform in order to improve the company profits, and the payment of royalty of Windows CE makes many manufacturers to far to catch up.

To sum up, each of the two kinds of operating system has their advantages and disadvantages. Windows CE has a good interactive interface, which is convenient for developing graphical applications, and the interoperability is strong; Windows CE adopt fully modular structure to design, which has strong commonality; a variety of development environments provided by Windows $\mathrm{CE}$ are similar that of Windows, the portability of them are strong. But the evil is that Windows CE system takes up too more memory resources, along with the improvement of hardware production and the fall of production cost, these shortcomings become no longer important, in view of the above features, Windows CE embedded operating system is chosen in this paper.

\section{THE DESIGN AND SET UP OF THE HARDWARE PLATFORM}

\subsection{Program Design Process}

After writing section data to SPTDATn register, if the ENSCK and MSTR of SPTDATn registers have been set, the SPI will begin to transfer. SPI operation card can be operated through the use of the typical program design process.

Program for the SPI module by following steps:

1. Set the baud rate to the prescaler registers (SPPREn).

2. Set SPCONn to configure the SPI module.

3. Write ten times of OxFF data to SPTDATn register to initialize the MMC or SD card.

4. Set a GPIO pin as nSS, lower the level to activate the MMC or SD card.

5. Tx data - check the situation of the ready flag bit to sign a $(\mathrm{REDY}=1)$, and then to write SPTDATn register data.

6. Rx data (1): TAGD bit of SPCONn is disabled for the standard model. Write 0xFF to SPTDATn register to ensure REDY is set, and then read data from cache.

7. Rx data (2): When SPCONn TAGD is enabled for Tx automatic garbage data model. Check whether REDY has been set up, and then read data from cache (then start automatically transmission).

8. Set a GPIO pin as nSS, higher the level, in order to disable MMC or SD card.

\subsection{Serial Flash Configuration Mode of SPI}

Serial Flash has the characteristics of less occupied of pin, which commonly uses serial peripheral interface such as SPI bus interface, etc., it is very appropriate as the data storage of system. The new FPGA products in XILINX are able to support the SPI interface [4] SPI bus complete the communication between main equipment and the dependent equipment under the SPI communication protocol using four signal lines, the typical SPI communication system contains one or more main equipment and at least one dependent device, the names and functions of the four SPI interface signals are as shown in Table 2. 
Table 2. The signal of SPI interface.

\begin{tabular}{|c|c|}
\hline SPI port signal name & Signal function description \\
\hline \hline SCLK & Serial clock of SPI port work \\
\hline MOSI & Data signal from main computer to department one, applying for sending executable code and data to department one \\
\hline MISO & Data signal from main computer to department one, applying for collecting data signal transferred by department one \\
\hline SS_n & Choose signal from computer piece, effective for low level. Abundon the control of department computer, and set MISO port at \\
high impedance
\end{tabular}

When there are only one master device and one dependent device, the communication interfaces are as shown in (Fig. 1). In this system, ARM is used as the main equipment, the FPGA as the department device, the ARM controls both the sequence in the process of communication through the SCLK signal, the SS $n$ is low, the ARM transfer configuration data to M25P16 this Flash chips through MOSI line. No matter the master, and dependent device, the configuration data is output when the system clock level jumps, and jump along the front at the next opposite level, and then is sent to another chip [5].

\subsection{Network Transmission Module}

To achieve the remote control network of the embedded devices, a standard Network communication interface need to be equipped in the process of network development.

In this paper, the system is access to Internet network, the corresponding Internet or network interface circuit is indis- pensable, however, there is no special Ethernet controller $53 \mathrm{C} 2440 \mathrm{~A}$ in the interior, need to mount an external bus through network controller to realize the need of S3C244OA connected to Ethernet. There are RTL8019、 RTL8029、CS8900、DM9000A, etc. as the common network chips in current market [6, 7].

This article chooses DM9000A as Ethernet physical layer interface. From (Fig. 2) we can learn that S3C2440A is access to Ethernet through DM9000A, the sent or received data are transformer into RJ45 interface or Ethernet controller after isolation and through transformation through Ethernet transformer HS9016. Here will connect DM9000A chip in the form of a 16 bit with $\mathrm{S} 3 \mathrm{C} 2440 \mathrm{~A}$ processor, connect 53C2440A data bus DATA0 DATA15 with chip SD0 SD15; and the address line ADDR2 is connected with chip CMD; Line selection nGCS3are connected to the nCS chip, nine extra interrupts are connected to the chip INT. The work base of DM9000A Ethernet controller is $0 \times 300$, and

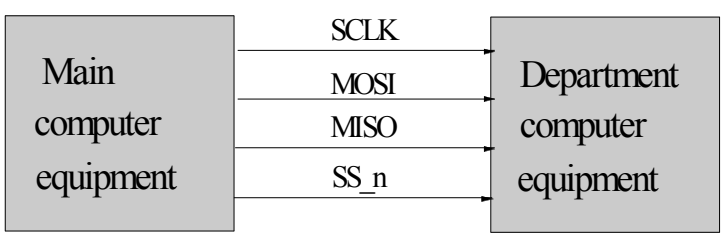

Fig. (1). Connection of SPI interface.

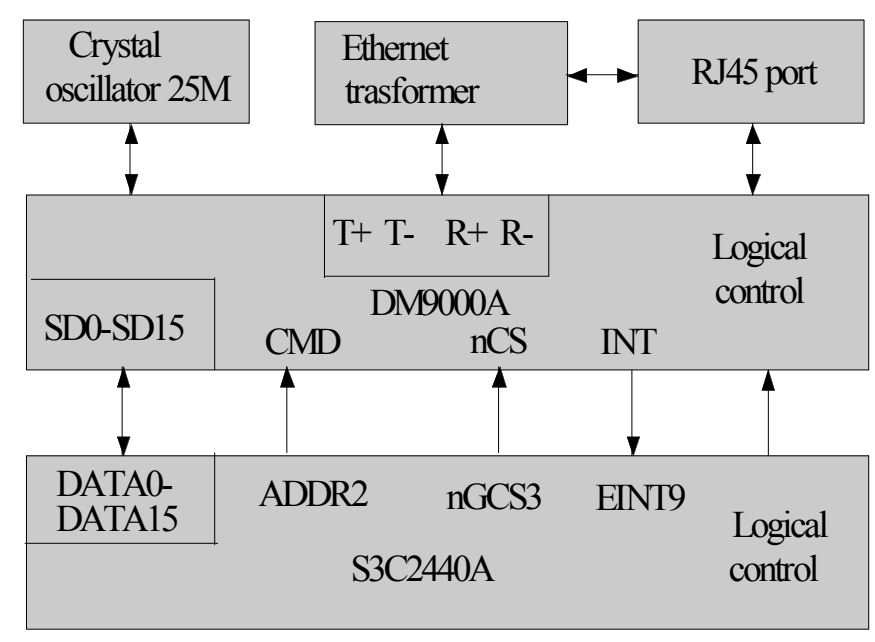

Fig. (2). Diagram of connection of S3C2440A and DM9000A. 
ADDR2 the address line of S3C2440A are connected to the chip/data port CMD, so to index port address is $0 \times 300$, data port operation address is $0 \times 304$.

\section{THE DESIGN OF THESOFTWARE SYSTEM}

Fedora is one of the many Linux released versions, compared with many other distributions such as Red Hat, CentOS, Ubuntu, the characteristics of Fedora are fast, stable and strong. It can be used, modified and distributed by anyone freely. Fedora is an operating system based on Red Hat Linux, and is gradually replacing the Red Hat Linux application in the personal field. At the same time, the application of Red Hat Linux in the field of business will be gradually replaced by Red Hat Enterprise Linux. Fedora is used as the test platform of the new technology, many new technologies are tested in the Fedora, if test results are stability, then the corresponding new technology will be put into the Red Hat Enterprise Linux $[8,9]$.

Fedora is active in the promotion of free software. At the same time, the installation of Fedora inherited many set tools such as interface Anaconda Red Hat Linux, so the software will not face too big differences after Fedora tests in being transplanted to RHEL, CentOS system, etc. So Fedora is chosen as Linux development platform [10].

\subsection{Connection of Windows Main Computers with ARM Development Board}

The resources of embedded ARM development board is limited, we will connect embedded Type ARM development board to the serial port and computer serial port through the embedded ARM development board with using Windows host serial port, if the computer doesn't has serial port, the U ports should be turned into serial ports, and the corresponding driver of $U$ port turn into serial port should be installed. If the TCP protocol is used, then we select the TCP/IP, internet access is used at this time. Serial port is used here, so corresponding COM3 should be chosen. Here The importance to be noted here that choosing COM should be according to the configuration of the "my computer", and the device manager in open COM port should be checked, haphazard selection should not happen, otherwise will lead to the useless of COM. Configuration the properties of $\mathrm{COM} 3$, the baud rate should be set at 115200 , and data is set at 8 , the parity bit should not be set, stop bit is set at 1 , data flow control bits are set zero. Set the super terminal properties, and the function keys, arrow keys, and Ctrl keys are set as the terminal keys [11].

\section{CONCLUSION}

Embedded system is decided according to specific requirements and they are very different. Embedded system has the requirements of little volume, low price and high reliability. It has been affecting and changing our lives in an unprecedented speed so that there are always embedded systems as far as we can see. Embedded system has capacious application areas; it has been used in the areas such as mobile phone, video camera, automotive electronics, industrial control equipment and medical devices. Although, no matter
IPC (Industrial Personal Computer) or SCM, none of them can satisfy the requirements of little volume, low price and high reliability of embedded system in depth. As the development of new techniques, the rapid expansion of mobile internet and the constantly reducing production cost, enhancing performance of integrated circuit chips, embedded system has ushered new expansion opportunity.

The application field of embedded technology has been wider in today's society, this paper puts forward a new way of configuration to the reconfiguration of FPGA based on ARM embedded technology, the FPGA can be separated from the direct link to the computer during reconfiguration.

This design builds the NFS file system, which makes the embedded Linux system connected with the remote Linux server, download configuration files from the Linux server to the ARM through ARM, and get the corresponding FPGA configuration file. Research and design the relevant circuit under SPI configuration mode, and connect ARM with the configuration circuit of FPGA. Choose main SPI Flash mode, set ARM as the main equipment, set the FPGA as dependent unit, and use ARM to transfer the configuration files to FPGA under the SPI communication protocol, to make FPGA reconfiguration.

In this design, there are still some places to be to improved continuously, such as experiments have been carried on just in FPGA of Spartan-3 without the other kinds of FPGA system tests. There are potential safety hazards during the process of transmission of FPGA configuration files, in the future design, the encryption and decryption functions of the FPGA configuration files can be increased in the system, to make remote configuration of FPGA system the safety performance higher, and promote the file processing method to various series of FPGA. In this way, as long as there are configurations files, ARM can be used on the configuration of FPGA, and there is no longer need of connection of the FPGA system with experimental computer.

\section{CONFLICT OF INTEREST}

The author confirms that this article content has no conflict of interest.

\section{ACKNOWLEDGEMENTS}

Declared none.

\section{REFERENCES}

[1] T. Sun, and W. Yuan, "Design of Embedded System and Develop Manual of Linux Drive-Some TARM9 Prosessor," $3^{\text {rd }}$ ed., Electronic Industry Press, Beijing, 2009.

[2] C. Zhou, and Y. Liu, "Analysis on ARM system and AMBA bus," Computer Engineering, no. 5, pp. 147-149, 2003.

[3] Y. Yan, W. Wu, and B. Zhang, "A network RTU based on embedded Linux," Electric Power Automation Equipment, vol. 24, no. 9, pp. 27-29, 2004.

[4] M. Zhen, "Real-time Data Acquisition and Processing System of USB Interface Based on FPGA," Ocean University of China, 2011.

[5] Q. Wen, and S. Chen, "Set and teaching of embedded direct course based on ARM system structure," Computer Education, no. 17, pp. 25-28, 2007. 
[6] B. Xi, and Y. Fang, "Research and implementation of network communication in embedded monitoring systera," Electric Power Automation Equipment, vol. 24, no. 7, pp. 68-71, 2004.

[7] B. StePhen, and S. Lanfear, "Linux future in the embedded systems market," Industry analysts, Venture Development Cooperation, vol. 6, pp. 67-71, 2002.

[8] "S3C2440A 32-Bit CMOS Microcontroller User's Manual," Revision 2 Samsung Electronics, 2008.
[9] L. Peter, and T. Mario, "Trends in embedded software engineer," IEEE Software, vol. 26, pp. 19-25, 2009.

[10] O' REILLY, "Linux Device Drivers" $3^{\text {rd }}$ ed., [s. 1.] : Octal Publishing, Inc, 2005.

[11] J. Chen, X. Li, and H. Zhang, "Design of Asynchronous serial communication based on ARM+Linux," Micro Computer Information, no. 08, pp. 139-156, 2009.

(C) Chun Zeng; Licensee Bentham Open.

This is an open access article licensed under the terms of the (https://creativecommons.org/licenses/by/4.0/legalcode), which permits unrestricted, non-commercial use, distribution and reproduction in any medium, provided the work is properly cited. 